\title{
Respiratory muscle aids to avert respiratory failure and tracheostomy: a new patient management paradigm
}

This article was published in the following Dove Press journal:

Journal of Neurorestoratology

21 February 2014

Number of times this article has been viewed

\author{
John R Bach ${ }^{1-3}$ \\ Anokhi D Mehta ${ }^{2}$ \\ 'Department of Physical Medicine \\ and Rehabilitation, ${ }^{2}$ Department of \\ Neurosciences, Rutgers-New Jersey \\ Medical School, Newark, NJ, USA; \\ ${ }^{3}$ Center for Ventilator Management \\ Alternatives, University Hospital, \\ Newark, NJ, USA
}

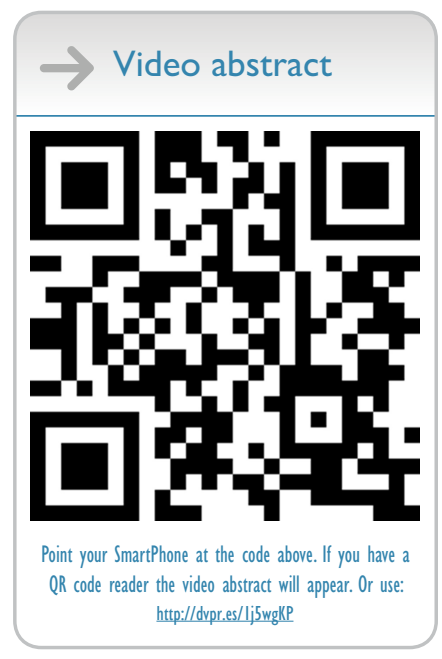

Correspondence: John R Bach Department of Physical Medicine and Rehabilitation, University Hospital B-403, I50 Bergen Street, Newark, NJ 07103, USA

Tel + I 9739722850

Fax + I 9739725725

Email bachjr@njms.rutgers.edu

\begin{abstract}
An April 2010 consensus of clinicians from 22 centers in 18 countries reported 1,623 spinal muscular atrophy type 1, Duchenne muscular dystrophy, and amyotrophic lateral sclerosis noninvasive intermittent positive pressure ventilatory support users, of whom 760 developed continuous dependence that prolonged their survival by more than 3,000 patientyears without tracheostomies. Four of the centers routinely extubated unweanable patients with Duchenne muscular dystrophy, so that none of their more than 250 such patients has undergone tracheotomy. This article describes the manner in which this is accomplished; that is, the use of noninvasive inspiratory and expiratory muscle aids to prevent ventilatory failure and to permit the extubation and tracheostomy tube decannulation of patients with no autonomous ability to breathe (ie, who are "unweanable" from ventilator support). Noninvasive airway pressure aids can provide up to continuous ventilatory support for patients with little or no vital capacity and can provide for effective cough flows for patients with severely dysfunctional expiratory muscles.
\end{abstract}

Keywords: glossopharyngeal breathing, assisted cough, neuromuscular disease, Duchenne muscular dystrophy, spinal muscular atrophy, amyotrophic lateral sclerosis

\section{Introduction}

Patients with progressive neuromuscular weakness typically develop acute respiratory failure (ARF) as a result of otherwise benign upper respiratory tract infections that develop into pneumonias and ARF as a result of an ineffective cough. Such patients can also develop $\mathrm{CO}_{2}$ narcosis as a result of supplemental $\mathrm{O}_{2}$ therapy without ventilatory assistance. They typically present to emergency services, are treated with oxygen and/or bilevel positive airway pressure at low spans that do not provide full ventilatory support or respiratory muscle rest, and are intubated for ARF. When they fail ventilator weaning trials and spontaneous breathing parameters or fail extubations to inadequate noninvasive ventilatory support and without mechanical cough assistance, they are told tracheotomy is their only option for survival. This article demonstrates that respiratory infections developing into pneumonia and ARF are avoidable and that even when intubation is required, ventilator weaning is not a prerequisite for extubation without undergoing tracheotomy. The respiratory muscle aids that permit patients to avoid tracheotomy can be introduced in outpatient clinics and in the home setting by specifically trained respiratory therapists, as well as in hospitals. The same aids can be used in critical care units to extubate and decannulate ventilator-dependent patients who are too weak to breathe on their own. 


\section{Pathophysiology}

Respiratory disability results either from primarily lung/ airways diseases, in which case arterial blood gas sampling and pulmonary function testing can be indicated and supplemental oxygen and bronchodilators can be beneficial, or from complications of respiratory muscle impairment. The former is characterized by hypoxia in the presence of eucapnia or hypocapnia until an exacerbation causes ARF. The latter is characterized by hypercapnia and hypoxemia caused by hypoventilation or respiratory insufficiency/failure resulting from an ineffective cough. The former is respiratory insufficiency/failure, whereas the latter may be ventilatory insufficiency/failure. Unfortunately, many physicians rarely distinguish between the two, referring to both, as well as evaluating and treating both, as respiratory insufficiency/ failure. This results in needless morbidity and mortality, not to mention cost and decreased quality of life. Symptomatic hypercapnic patients benefit from noninvasive intermittent positive pressure ventilatory support (NVS) for at least part of the day, most often overnight. With progressive inspiratory muscle weakness, ventilator-free breathing ability is eventually lost.

Ventilatory insufficiency/failure can be nocturnal only and result from diaphragm dysfunction, with the patient unable to breathe when supine; it can be from total inspiratory muscle failure; it can result from inadequate central ventilatory drive; or it can result from severe obesity or chest wall restriction. Many patients with ventilatory insufficiency survive for years without ventilator use at the cost of orthopnea and increasing hypercapnia, with its associated symptoms and danger of $\mathrm{CO}_{2}$ narcosis. Bicarbonate retention by the kidneys compensates for the effect of $\mathrm{CO}_{2}$ retention on blood $\mathrm{pH}$, but this also depresses central ventilatory drive and allows the brain to accommodate to hypercapnia without overt symptoms of acute ventilatory failure. Supplemental oxygen therapy instead of ventilatory assistance further exacerbates hypercapnia via the Haldane effect, by which the oxygenation of hemoglobin promotes dissociation of $\mathrm{H}^{+}$ from hemoglobin, shifting the bicarbonate buffer equilibrium toward $\mathrm{CO}_{2}$ formation and thereby releasing $\mathrm{CO}_{2}$ from red blood cells to exacerbate hypercapnia. It is not uncommon for the hypercapnia associated with supplemental oxygen therapy to result in $\mathrm{CO}_{2}$ narcosis and ventilatory arrest. When symptomatic, hypercapnic patients are properly treated with NVS, blood gases normalize, symptoms resolve, and the kidneys excrete the excess bicarbonate ions. Because of the need to take bigger breaths to maintain normal carbon dioxide tension and blood $\mathrm{pH}$ when discontinuing NVS in the morning, dys- pnea can necessitate increasing periods of daytime NVS until patients require it continuously (CNVS). Some patients with ventilatory muscle failure and no measurable vital capacity (VC) with their respiratory muscles use only nocturnal aid and rely on glossopharyngeal breathing (GPB), an autonomous method of assisting respiratory muscle function, to ventilate their lungs during daytime hours.

There are three respiratory muscle groups: the inspiratory muscles, the expiratory (predominantly abdominal and upper chest wall) muscles for coughing, and the bulbar-innervated muscles to protect the airways. Although the inspiratory and expiratory muscles can be completely supported, such that even patients with $0 \mathrm{~mL}$ VC have used CNVS for up to 60 years without resort to tracheostomy (Figure 1), there are no effective noninvasive measures to assist bulbarinnervated muscle function. Thus, the only indication for tracheotomy in an unweanable patient is the aspiration of saliva to the degree that the oxyhemoglobin saturation $\left(\mathrm{SpO}_{2}\right)$ decreases and remains below 95\%. ${ }^{1}$ Fortunately, the only neuromuscular disease (NMD) in which this happens is in advanced bulbar amyotrophic lateral sclerosis (ALS) after patients have often entirely lost the ability to speak and

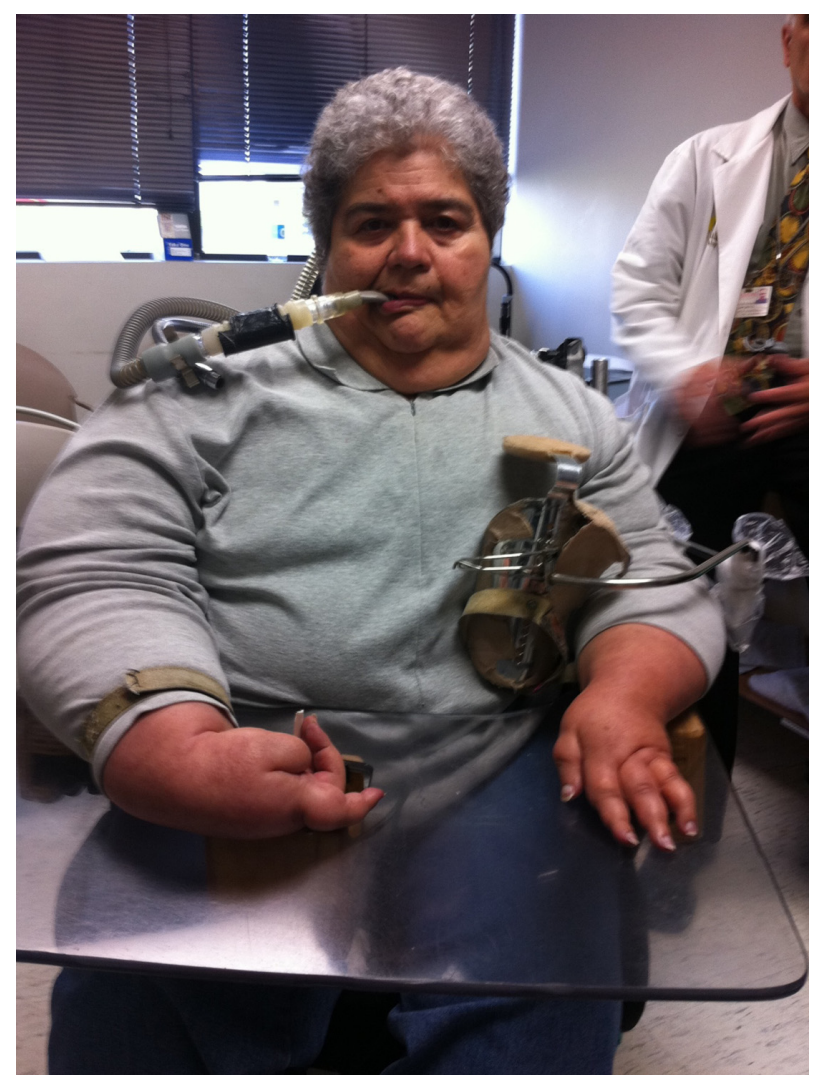

Figure I Postpolio survivor dependent on continuous noninvasive intermittent positive pressure ventilatory support (CNVS), including daytime mouth piece CNVS, for 59 years. 
swallow food. Such patients develop essentially irreversible upper airway obstruction and require tracheostomy tubes to protect the airways. Spinal muscular atrophy type 1 (SMA1) survival into adulthood is possible (Figure 2), using both CNVS and continuous tracheostomy mechanical ventilation (CTMV); however, tracheostomy-ventilated patients have greater levels of ventilator dependence ${ }^{2}$ and reduced verbal abilities, and up to $80 \%$ die from complications of the tube. ${ }^{3}$

\section{Patient evaluation}

Patients with diminished ventilatory reserve who are able to walk commonly complain of exertional dyspnea. Eventually, arterial $\mathrm{CO}_{2}$ increases and results in morning headaches, fatigue, sleep disturbances, and hypersomnolence. ${ }^{4}$ For wheelchair users, symptoms may be minimal except during intercurrent respiratory infections, when they complain of anxiety, inability to fall asleep, and dyspnea. Patients are observed for tachypnea, paradoxical breathing, hypophonia, nasal flaring, use of accessory respiratory musculature, cyanosis, flushing or pallor, and airway secretion congestion. Lethargy and confusion signal $\mathrm{CO}_{2}$ narcosis.

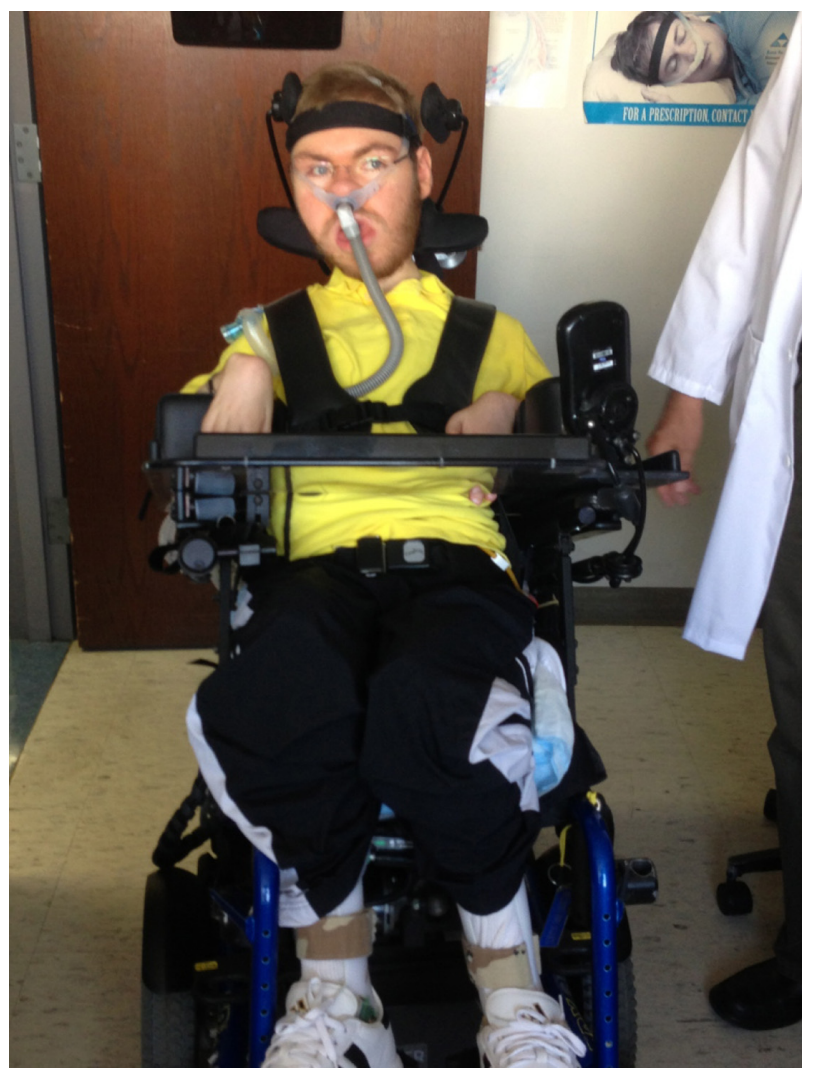

Figure 2 Twenty-one-year-old man with typical spinal muscular atrophy type I who has been using noninvasive intermittent positive pressure ventilatory support since the age of I year old and is now continuously dependent.
Evaluation necessitates four items: a spirometer, peak flow meter, capnograph, and oximeter. The VC is measured in sitting and supine positions. The VC difference should be less than 7\%. Because hypoventilation is worse during sleep, supine, rather than sitting position, VC is the most important indicator of ventilatory dysfunction. When the sitting-supine VC difference is greater than $20 \%$, orthopnea can signal the need for nocturnal NVS. Patients wearing thoracolumbar bracing should have the VC measured both with the brace on and off, as a good-fitting brace can increase $\mathrm{VC}$ and a poorly fitting one can decrease it. Spirometry is also useful for monitoring progress with GPB and air stacking; that is, retention of a maximum lung volume of aid delivered by manual resuscitator or volume cycling ventilator that can be held by the glottis. The maximum volume is termed the maximum insufflation capacity (MIC). The extent to which the MIC exceeds VC (MIC - VC) objectively quantitates glottic, and therefore bulbar-innervated, muscle integrity. Patients who learn GPB can often air-stack consecutive GPB gulps to or beyond the MIC. ${ }^{4}$ A nasal interface or lip seal can be used for air stacking when the lips are too weak for effective air stacking via the mouth.

Cough peak flows (CPF) are measured using a peak flow meter (Access Peak Flow Meter; Healthscan Products Inc., Cedar Grove, NJ, USA). CPF of $160 \mathrm{~L} /$ minute are the minimum needed to cough effectively, ${ }^{5}$ and this is the best indicator for tracheostomy tube removal irrespective of pulmonary function. Indeed, $40 \%$ of patients with ALS can survive despite continuous ventilator dependence, using strictly noninvasive aids. ${ }^{1}$ Patients with VCs less than 1,500 $\mathrm{mL}$ have assisted CPF measured from a maximally stacked volume of air and with an abdominal thrust delivered simultaneously with glottic opening. ${ }^{6}$ Coughing from a deep air-stacked volume with a concomitantly applied abdominal thrust is termed a manually assisted cough.

For the stable patient without intrinsic pulmonary disease, arterial blood gas sampling is unnecessary. In addition to the discomfort, $25 \%$ of patients hyperventilate as a result of anxiety and pain during the procedure. ${ }^{7}$ Noninvasive continuous blood gas monitoring; that is, monitoring endtidal $\mathrm{CO}_{2}$ (capnography) and oximetry, yield more useful information, particularly when performed continuously during sleep at home.

Although all clearly symptomatic patients with diminished lung volumes require a trial of NVS to ease symptoms, if symptoms are questionable, nocturnal noninvasive blood gas monitoring can be performed. The oximeter and the capnograph, which measures end-tidal $\mathrm{pCO}_{2}$, must be capable of 
collating the data. ${ }^{4}$ These studies are most conveniently performed in the home. Any questionably symptomatic patient with decreased VC, multiple nocturnal oxyhemoglobin desaturations below 95\%, and elevated nocturnal carbon dioxide tension should undergo a trial of nocturnal NVS. Because, in general, only patients improperly treated with supplemental $\mathrm{O}_{2}$ develop $\mathrm{CO}_{2}$ narcosis, and ARF is generally caused by ineffective airway secretion management, any patient finding that NVS use is more burdensome than symptoms of ventilatory insufficiency can discontinue NVS and return for a reevaluation in 3-6 months.

For symptomatic patients with normal VC, an unclear pattern of oxyhemoglobin desaturation, and no apparent carbon dioxide retention, sleep disordered breathing is suspected and a polysomnogram is warranted. ${ }^{8}$ Polysomnography is not indicated for patients with decreased VC (NMD) because it is programmed to interpret every apnea and hypopnea as resulting from central or obstructive events rather than from inspiratory muscle weakness. Further, treatment of asymptomatic NMD patients on the basis of polysomnographic abnormalities neither prolongs life nor improves its quality.

\section{Physical medicine respiratory muscle aids}

Inspiratory and expiratory muscle aids are devices and techniques that involve either the manual or mechanical application of forces to the body or intermittent pressure changes to the airway to assist inspiratory or expiratory muscle function. The devices that act on the body include body ventilators that create pressure changes around the thorax and abdomen. Negative pressure applied to the airways during expiration assists coughing, just as positive pressure applied to the airways during inhalation (NVS) assists the inspiratory muscles. Continuous positive airway pressure does not assist ventilation and is not useful for patients with primarily ventilatory impairment.

\section{GPB}

Both inspiratory and indirectly, expiratory muscle function can be assisted by GPB. ${ }^{9}$ GPB can provide an individual with weak inspiratory muscles and no $\mathrm{VC}$ or breathing tolerance with normal alveolar ventilation when not using a ventilator or in the event of sudden ventilator failure, day or night. ${ }^{9,10}$ The technique involves the use of the glottis to add to an inspiratory effort by pistoning boluses of air into the lungs. The glottis closes with each bolus. One breath usually consists of six to nine boluses of 40-200 mL each (Figure 3). During the training period, the efficiency of GPB can be monitored by spirometrically measuring the milliliters of air per gulp, gulps per breath, and breaths per minute. A training manual $^{11}$ and numerous videos are available. ${ }^{12,13}$

Although severe oropharyngeal muscle weakness can limit the usefulness of GPB, we have managed 13 Duchenne muscular dystrophy (DMD) ventilator users who had no breathing tolerance other than by GPB. ${ }^{14}$ Approximately $60 \%$ of ventilator users with no autonomous ability to breathe and good bulbar muscle function can use GPB and discontinue ventilator use for up to all day. ${ }^{9,15}$ GPB is rarely useful in the presence of an indwelling tracheostomy tube. The safety and versatility afforded by GPB are additional reasons to eliminate tracheostomy in favor of noninvasive aids.

Because of their generally intact bulbar musculature, high-level spinal cord injury (SCI) patients are ideal candidates to master GPB for ventilator-free breathing and be decannulated to NVS. In some centers, these patients are decannulated to free them from the fear of ventilator failure or accidental ventilator disconnection. Respiratory management of high-level SCI patients as a function of spinal cord level is seen in Table 1.9,15

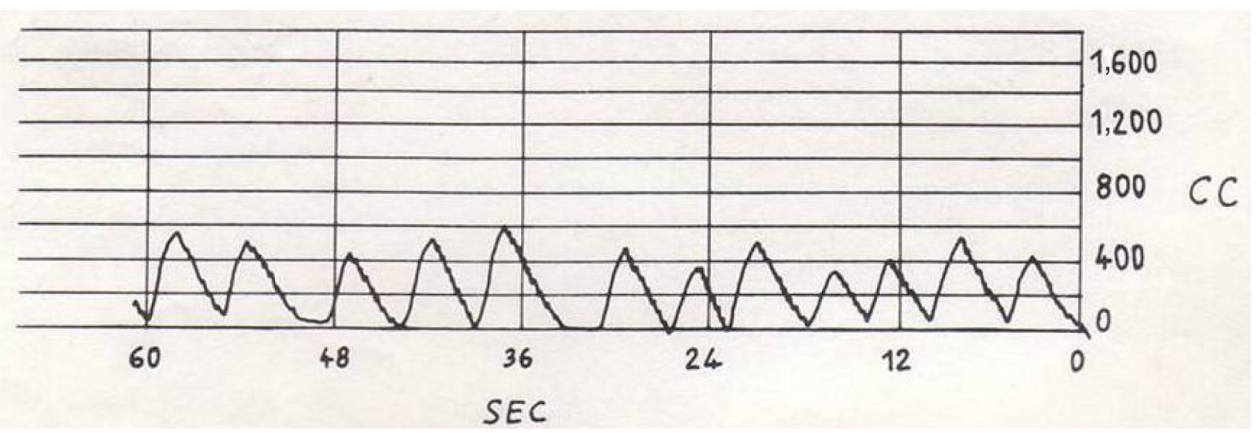

Figure 3 Normal minute ventilation (60-90 mL/gulp, 6-8 gulps per breath, 12 breaths per minute) throughout daytime hours by glossopharyngeal breathing for an individual with no measurable vital capacity for 52 years. Maximum glossopharyngeal single-breath capacities can exceed $3,000 \mathrm{~mL}$ for many such individuals. 
Table I Management of patients with spinal cord injury

\begin{tabular}{lllll}
\hline Level* & VC, $\mathbf{m L}$ & $\begin{array}{l}\text { Bulbar function/ } \\
\text { neck function** }\end{array}$ & Daytime & Nocturnal \\
\hline Above & 0 & $\begin{array}{l}\text { Inadequate/ } \\
\text { inadequate }\end{array}$ & TIPPV & TIPPV \\
Cl & & $\begin{array}{l}\text { Adequate/inadequate } \\
\text { C2-C3 }\end{array}<200$ & EPR & N/MIPPV \\
Below & $>200$ & Adequate/adequate & MIPPV/IAPV & N/MIPPV \\
C2 & & & & \\
\hline
\end{tabular}

Notes: *Motor levels; **adequate neck function involves sufficient oral and neck muscular control to rotate, flex, and extend the neck to grab and use a mouthpiece for IPPV and adequate bulbar function to prevent aspiration of saliva to the degree that the oxyhemoglobin saturation baseline decreases below $95 \%$.

Abbreviations: VC, vital capacity; TIPPV, tracheostomy intermittent positive pressure ventilation; EPR, electrophrenic pacing; MIPPV, mouthpiece intermittent positive pressure ventilation; NIPPV, nasal intermittent positive pressure ventilation; IAPV, intermittent abdominal pressure ventilator.

\section{Intervention objectives}

The intervention goals are to maintain lung and chest wall compliance and to promote normal lung and chest wall growth for children, to maintain normal alveolar ventilation around the clock, and to maximize $\mathrm{CPF}$.

\section{Long-term management Goal one: maintain pulmonary compliance, lung growth, and chest wall mobility}

Pulmonary compliance diminishes when a patient cannot expand the lungs to predicted inspiratory capacity. As the VC decreases, the largest breath one can take only expands a fraction of lung volume. Similar to limb articulations, regular mobilization is required to prevent chest wall contractures and lung restriction. This can only be achieved by providing deep insufflations, air stacking, or nocturnal NVS. ${ }^{16} \mathrm{We}$ recommend daily air stacking once the $\mathrm{VC}$ is less than $80 \%$ of normal. Patients who cannot close the glottis and, therefore, cannot air stack must be passively insufflated using a CoughAssist ${ }^{\mathrm{TM}}$ (Respironics International Inc., Murrysville, PA, USA) pressure-cycling ventilator at pressures of 40-70 $\mathrm{cm} \mathrm{H}_{2} \mathrm{O}$, or a manual resuscitator with the exhalation valve blocked. The maximum passive insufflation volume can be termed the "lung insufflation capacity."17

The primary objectives of lung expansion therapy are to increase the $\mathrm{VC}$ and to maximize $\mathrm{CPF}$, to maintain or improve pulmonary compliance, to diminish atelectasis, and to master NVS. The deep lung volumes by air stacking also permit patients to raise voice volume and speak longer phrases. Because any patient who can air stack is also able to use NVS, if such a patient is intubated for ARF, he or she can more easily be extubated directly to CNVS, regardless of ventilator-free breathing ability. Patients are instructed to air stack 10-15 times at least two or three times daily, usually using a manual resuscitator. Because of the importance of air stacking, NVS is provided via ventilators using volume, rather than pressure cycling, on assist/control mode.

Infants cannot air stack or cooperate with passive insufflation therapy. All babies with SMA1 and children with SMA2 or other conditions who have paradoxical chest wall movement require nocturnal NVS to prevent pectus excavatum and promote lung growth, as well as for ventilatory assistance..$^{18}$ In addition to nocturnal aid, deep insufflations may be possible by delivering air from a manual resuscitator via an oral-nasal interface and timing the air delivery to the child's breathing. Children can become cooperative with deep insufflation therapy by 14-30 months of age.

\section{Goal two: maintain normal alveolar ventilation by inspiratory muscle assistance}

Although the inspiratory muscles can be assisted by applying pressures to the body, negative-pressure body ventilators cause obstructive apneas, are less effective than NVS, and become increasingly less effective with age and decreasing pulmonary compliance. ${ }^{19}$ Blood gases improve dramatically when switching patients to NVS.

A body ventilator that continues to be useful is the intermittent abdominal pressure ventilator, or Exsufflation Belt ${ }^{\mathrm{TM}}$ (Respironics International Inc., Murrysville, PA, USA). It involves the intermittent inflation of an elastic air sac that is contained in a corset or belt worn beneath the patient's outer clothing (Figure 4). The sac is cyclically inflated by a positive pressure ventilator. Bladder inflation moves the diaphragm

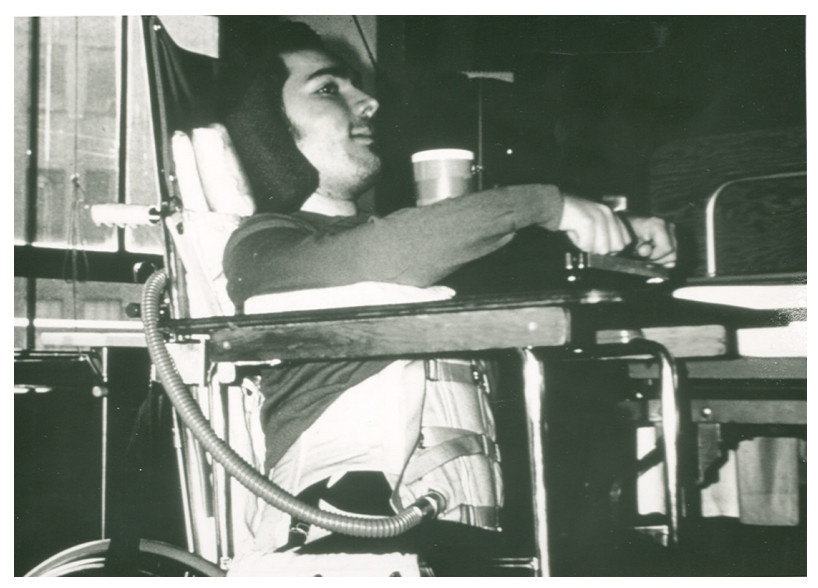

Figure 4 A 44-year-old with Duchenne muscular dystrophy, using an intermittent abdominal pressure ventilator (Exsufflation Belt ${ }^{\mathrm{TM}}$; Respironics International Inc., Murrysville, PA, USA), during daytime hours, and lip-seal ventilation nightly for 19 years. The air bladder inside the girdle is connected to the ventilator circuit (seen here), and then the girdle is placed under the clothes and over the patient's abdomen. 
upward to assist in expiration. During bladder deflation, gravity causes the abdominal contents and diaphragm to return to the resting position, and inspiration occurs passively. A trunk angle of $30^{\circ}$ or more from the horizontal is necessary for it to be effective. If the patient has any inspiratory capacity or is capable of GPB, he or she can add volumes of air to that taken in mechanically. The intermittent abdominal pressure ventilator augments tidal volumes by from $300 \mathrm{~mL}$, to as much as 1,200 $\mathrm{mL}$; patients with less than 1 hour of breathing tolerance usually prefer it to using NVS during daytime hours. $^{20}$

\section{NVS}

NVS should be offered to patients who are symptomatic for hypoventilation. It can become critical once hypoventilation causes the $\mathrm{SpO}_{2}$ baseline to decrease to less than 95\%. NVS is delivered via lip seal, nasal, and oral-nasal interfaces for nocturnal ventilatory support. Mouthpiece/lip cover and nasal NVS are open systems that require the user to rely on central nervous system reflexes to prevent excessive insufflation leakage during sleep. ${ }^{4,21}$ We recommend assist control volume cycling at $800-1,500 \mathrm{~mL}$, with an active ventilator circuit. These volumes permit patients to vary tidal volumes and best rest inspiratory muscles, ${ }^{22}$ and permit patients to air stack to increase cough flows overnight without assistance. Supplemental oxygen and sedatives can render NVS ineffective. NVS can be introduced in the clinic, home, or hospital setting.

There are numerous commercially available nasal interfaces (eg, continuous positive airway pressure masks). Several should be tried and the patient encouraged to alternate their use. Excessive insufflation leakage can be avoided by switching to the use of a closed, noninvasive system, such as a lip seal-nasal prong system. Such interfaces deliver air via mouth and nose during sleep and require minimal strap pressure. This optimizes skin comfort and minimizes air (insufflation) leakage. Excessive leakage is also prevented by sustaining ventilatory drive by maintaining normal daytime $\mathrm{CO}_{2}$ and avoiding supplemental $\mathrm{O}_{2}$ and sedatives.

NVS via a $15 \mathrm{~mm}$ angled mouthpiece is the most important method of daytime ventilatory support. Some patients keep the $15 \mathrm{~mm}$ angled mouthpiece between their teeth all day. ${ }^{23}$ Most have the mouthpiece fixed near the mouth. A metal clamp attached to a wheelchair can be used for this purpose, or the mouthpiece can be fixed onto motorized wheelchair controls, most often sip and puff, chin, or tongue controls. The Trilogy ${ }^{\mathrm{TM}}$ ventilator (Philips Respironics Inc., Murrysville, PA, USA) is now available with an attached flexible support arm that can hold a mouthpiece adjacent to the NVS user's mouth (Figure 5). The ventilator is also set for large tidal volumes, often $800-1,500 \mathrm{~mL}$, for daytime support. The patient grabs the mouthpiece with his mouth, triggering air delivery. Simply touching the mouthpiece triggers it to supplement or substitute for inadequate autonomous breath volumes. The patient varies the volume of air taken from ventilator cycle to ventilator cycle and breath to breath to vary speech volume and cough flows, as well as to air stack for lung volume recruitment. Some neck movement and lip function are needed to grab the mouthpiece and use it without leaking air. The soft palate must move in the posteriocranial direction to seal off the nasopharynx. In addition, the patient must open the glottis and vocal cords, dilate the hypopharynx, and maintain airway patency. These normally reflex movements can take time to relearn for CTMV users before decannulation to CNVS. ${ }^{24}$

Nasal NVS is most practical for use during sleep, but it is also indicated for infants and for those who cannot grab or retain a mouthpiece because of oral muscle weakness, inadequate jaw opening, or insufficient neck movement.

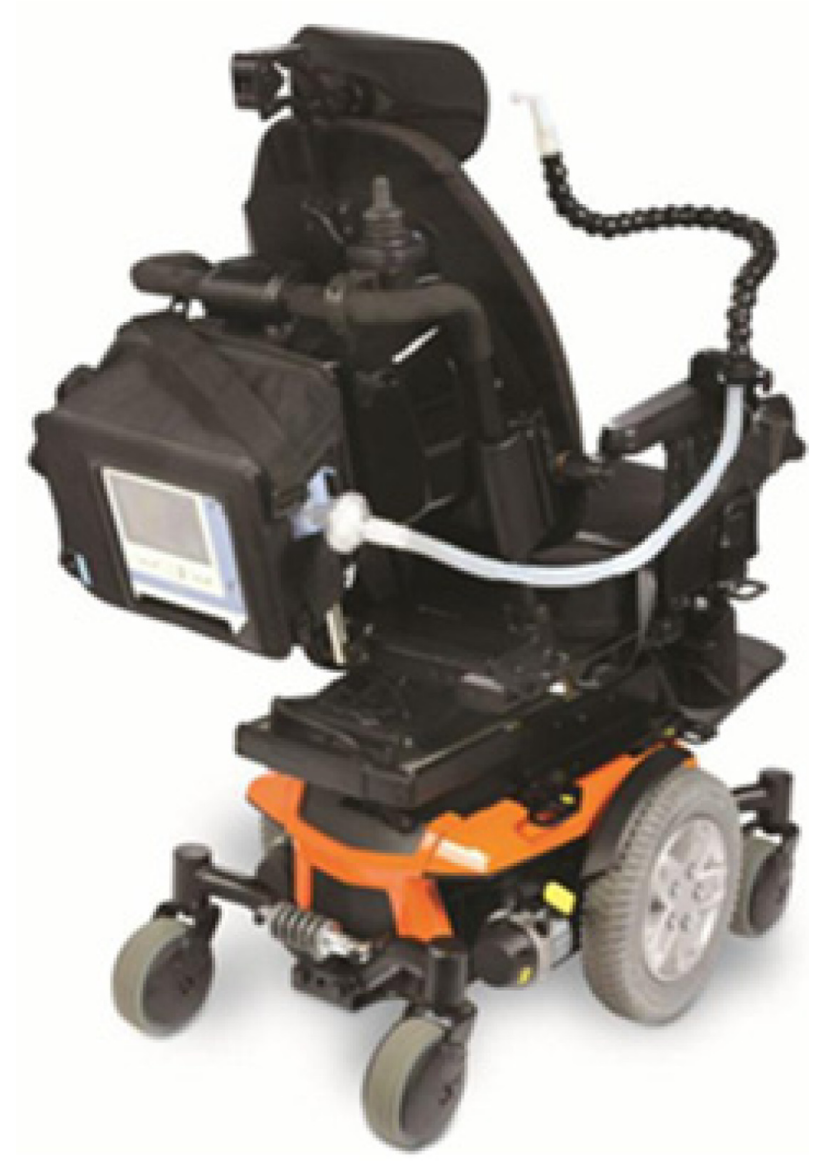

Figure 5 Support arm on the Trilogy ventilator (Philips Respironics Inc., Murrysville, PA, USA) to hold a mouthpiece for daytime ventilatory support. 
Daytime nasal CNVS is also a viable and desirable alternative to tracheostomy. ${ }^{3}$ Nasal NVS users learn to close their mouths or seal off the oropharynx with their soft palates and tongues to prevent oral insufflation leakage.

Suboptimal humidification dries out and irritates nasal mucous membranes, causes sore throat, and results in vasodilatation and nasal congestion. Increased airflow resistance to $8 \mathrm{~cm} \mathrm{H}_{2} \mathrm{O} / \mathrm{L}$ per second can be caused by the loss of humidity that is caused by unidirectional airflow with expiration via the mouth during nasal NVS. ${ }^{25}$ This can be reduced by humidifying the air, using a hot water bath humidifier. ${ }^{25}$ Decongestants can relieve sinus irritation and nasal congestion. Switching to a lip cover-only interface can relieve most, if not all, difficulties associated with nasal NVS.

Abdominal distention tends to occur sporadically in NVS users. The air usually passes as flatus once the patient is mobilized in the morning. When severe, however, it can increase ventilator dependence. Patients using volume cycling during sleep can be switched to pressure cycling to decrease abdominal distention. Sometimes a rectal tube can be helpful to decompress the colon or a nasogastric or gastrostomy tube used to burp out the air. Open gastrostomy, ${ }^{26}$ radiographically inserted gastrostomy, and percutaneous endoscopic gastrostomies with a hole created in the ventilating oronasal interface to pass the tube, can all be performed safely without airway intubation for patients with little or no autonomous breathing ability.

Despite aggressive lung mobilization and expansion three times daily, often to pressures more than $80 \mathrm{~cm} \mathrm{H}_{2} \mathrm{O}$, and along with CNVS for more than 50 years in many cases, we have had a single case of pneumothorax in more than 1,000 NVS users. Although often described as a complication or limiting factor for NVS, secretion encumbrance results from failure to use assisted coughing or mechanical insufflation-exsufflation (MI-E).

In SCI, CNVS is an alternative to electrophrenic and diaphragm pacing and tracheostomy for CTMV. Other than for uncontrollable seizures and inability to cooperate, there are no contraindications to long-term use of NVS. ${ }^{27}$

\section{Goal three: assist expiratory muscles to augment cough flows}

Manually assisted coughing is the use of air stacking and then an abdominal thrust timed to glottic opening. With the higher lung volumes by air stacking, assisted CPF of $255 \pm 100 \mathrm{~L} /$ minute were obtained by comparison with $150 \pm 120 \mathrm{~L} /$ minute unassisted. ${ }^{6}$ This is the difference between coughing effectively to prevent pneumonia and
ARF or not. ${ }^{28}$ The inability to generate $160 \mathrm{~L} /$ minute of assisted CPF indicates upper-airway obstruction or severe bulbar-innervated muscle dysfunction and indicates need for evaluation by laryngoscopy for reversible lesions.

Mechanically assisted coughing is the use of MI-E (CoughAssist ${ }^{\mathrm{TM}}$; Philips Respironics Inc., Murrysville, PA, USA), with or without an exsufflation-timed abdominal thrust. Deep insufflations followed immediately by deep exsufflations at pressures of 40 to $-40 \mathrm{mmHg}\left(54.1 \mathrm{~cm} \mathrm{H}_{2} \mathrm{O}\right)$ are usually the most effective and preferred. The MI-E can be provided via an oral-nasal mask, a simple mouthpiece, or a translaryngeal or tracheostomy tube, in which case there is a considerable pressure drop-off and diminution of air flows, ${ }^{29}$ but it is still effective in clearing airway secretions and preparing patients for extubation or decannulation. ${ }^{30}$ When delivered via the latter, the cuff, when present, should be inflated and pressures of $50-70 \mathrm{~cm} \mathrm{H}_{2} \mathrm{O}$ used. The CoughAssist $^{\mathrm{TM}}$ can be manually or automatically cycled. Manual cycling facilitates caregiver-patient coordination of inspiration and expiration with insufflation and exsufflation. One treatment consists of about five cycles of MI-E followed by a short period of normal breathing or ventilator use to avoid hyperventilation. Insufflation and exsufflation times are adjusted to provide maximum chest expansion and rapid lung emptying. In general, 2-4 seconds are required. Treatment continues until no further secretions are expulsed and secretion-related oxyhemoglobin desaturations are reversed. Use can be required as frequently as every 30 minutes around the clock during chest infections.

The use of MI-E via the upper airway can be effective for children as young as 11 months of age. Patients this young can become accustomed to MI-E and permit its effective use by not crying or closing the glottis. Between 2.5 and 5 years of age, most children cooperate and cough on cue. Exsufflation-timed abdominal thrusts are also used for infants.

Whether via the upper airway or via indwelling airway tubes, routine airway suctioning misses the left main stem bronchus about $90 \%$ of the time. ${ }^{31}$ MI-E provides the same exsufflation flows in both left and right airways without the discomfort or airway trauma of tracheal suctioning. Patients prefer MI-E to suctioning for comfort and effectiveness, and they find it less tiring. ${ }^{32}$ Deep suctioning, whether via airway tube or via the upper airway, can be discontinued for most patients.

The VC, pulmonary flow rates, and $\mathrm{SpO}_{2}$, when abnormal, improve with clearing of airway secretions and mucus by MI-E. ${ }^{33}$ An increase in VC of $15 \%-42 \%$ was noted 
immediately after treatment in 67 patients with "obstructive dyspnea," and a 55\% increase in VC was noted after MI-E in patients with neuromuscular conditions with no adverse hemodynamic effects. ${ }^{34} \mathrm{We}$, too, have observed $15 \%-400 \%$ (200-800 mL) improvements in VC and normalization of $\mathrm{SpO}_{2}$ as MI-E eliminates airway mucus for NVS users with chest infections. ${ }^{35}$

Of the three muscle groups required for effective coughing, MI-E can only take the place of the inspiratory and expiratory muscles. Thus, it cannot be used to avert tracheotomy very long in the presence of airway collapse, glottis spasticity, or continuous saliva aspiration, as often becomes the case in advanced bulbar ALS. In contrast, patients with completely intact bulbar muscle function, such as most ventilator users with traumatic tetraplegia, can usually air stack to volumes of $3 \mathrm{~L}$ or more, and unless the patient is very scoliotic or obese, a properly delivered abdominal thrust can result in manually assisted CPF of 350-540 L/minute. These flows should be more than adequate to clear the airways and prevent pneumonia and ARF without need for MI-E. Thus, the patients who benefit most from MI-E have assisted CPF less than $300 \mathrm{~L} /$ minute. Patients with DMD and other myopathies benefit greatly from MI-E. ${ }^{28}$ Patients with respiratory muscle weakness complicated by scoliosis and an inability to capture the asymmetric diaphragm by abdominal thrusting also benefit greatly from MI-E.

\section{Oximetry feedback protocol}

For a hypercapnic patient with desaturation resulting from chronic alveolar hypoventilation or the patient being switched from CTMV, introduction to and use of mouthpiece or nasal NVS is facilitated by oximetry feedback. An $\mathrm{SpO}_{2}$ alarm set at $94 \%$ signals the patient to normalize alveolar ventilation and $\mathrm{SpO}_{2}$ and, by taking deeper breaths, maintain $\mathrm{SpO}_{2}$ higher than $94 \%$ all day. ${ }^{28}$ When it is no longer possible to achieve this by unassisted breathing, it is done by mouthpiece or nasal NVS. With time, the patient requires increasing periods of NVS to maintain alveolar ventilation. In this manner, central ventilatory drive can be reset.

Continuous $\mathrm{SpO}_{2}$ feedback is especially important during respiratory tract infections. The cough flows of infants and small children who never have the strength to sit are inadequate to prevent chest cold-triggered pneumonias and ARF. The patients use MI-E for any dip in $\mathrm{SpO}_{2}$ below 95\%. When using CNVS, dips are usually caused by bronchial mucous plugging that, if not quickly cleared, can cause atelectasis, pneumonia, and collapsed airways. Thus, patients are instructed to use NVS and MI-E to maintain normal $\mathrm{SpO}_{2}$ to avert pneumonia, ARF, and hospitalization. For adults with infrequent chest colds, rapid access to MI-E may be all that is necessary.

\section{Invasive ventilatory support}

The use of noninvasive aids can be contraindicated by the presence of depressed cognitive function, orthopedic conditions interfering with noninvasive interface use, pulmonary disease necessitating a high fraction of inspired oxygen, or uncontrolled seizures or substance abuse. ${ }^{36}$ Although CTMV can extend survival for NMD patients, ${ }^{37}$ morbidity and mortality outcomes are not as favorable as with CNVS. ${ }^{38,39}$ Tracheotomy is indicated for severe bulbar ALS patients $^{1}$ and the occasional SMA1 patient. ${ }^{2}$

\section{Long-term outcomes}

Considering SMA, we reported 27 patients using CTMV (mean age, 78.2 months; range, 65-179 months). Twenty-five of 27 lost all autonomous breathing ability immediately on tracheotomy. None of the 21 who had not developed the ability to verbalize before undergoing tracheotomy did so after tracheotomy. In contrast, 72 SMA1 patients using NVS are alive at a mean age of 86.1 months (range, 13-196 months); 13 died at age 52.3 months (range, 13-111 months). Sixty-seven of the 75 could communicate verbally. Fifteen SMA1 patients are now older than 10 years, and six are older than 15 years, without tracheostomy tubes and despite requiring CNVS in most cases. ${ }^{2,40}$ Others have also reported CNVS dependence for patients with SMA1. ${ }^{41}$

Considering DMD, 101 of our nocturnal-only NVS users eventually became CNVS-dependent for from 7.4 \pm 6.1 years to $30.1 \pm 6.1$ years of age, with 56 patients still alive. Twenty-six of the 101 became CNVS dependent without hospitalization. Eight CTMV users were decannulated to CNVS. Forty-five consecutive intubated DMD patients who could not pass spontaneous breathing trials before or after extubation were successfully extubated to CNVS and MI-E. Seven have lived to older than 40 years, including four who have required CNVS for 28, 19, 21, and 24 years to ages 41, 44,48 , and 47 years. Others have also reported prolongation of life for DMD by CNVS. ${ }^{42-44}$ In a controlled study of 109 DMD CNVS-dependent patients, the 88 CNVS users survived 10 years longer than those dependent on CTMV. ${ }^{45}$ CNVS is also an alternative to intubation in the perioperative management of children with flaccid neuromuscular scoliosis. ${ }^{46}$

Considering ALS, of 176 patients using nocturnal NVS, $109(42 \%)$ went on to require CNVS for about 10 months 
before their $\mathrm{SpO}_{2}$ baseline decreased below 95\% because of saliva aspiration resulting from bulbar-innervated muscle impairment. Diaphragm pacing does not increase survival or provide any ventilatory assistance or support for patients with ALS or any other NMD. ${ }^{47}$

\section{Extubation of unweanable patients}

Specific extubation criteria and a new extubation protocol were developed for patients with respiratory muscle inadequacy (Table 2). Once meeting the criteria, oro- or nasogastric tubes are removed to facilitate postextubation nasal NVS. The patient is then extubated directly to NVS on assist/control 800-1,500 mL (rate, 10-14 per minute in ambient air). The NVS is delivered via nasal, oral-nasal, and mouthpiece interfaces. Patients keep $15 \mathrm{~mm}$ angled mouthpieces accessible (Figure 1) and wean themselves, when possible, by taking fewer and fewer intermittent positive pressure ventilations, as tolerated. Diurnal nasal NVS is used for those who cannot secure the mouthpiece. They use nasal or oronasal interfaces for nighttime ventilation. For episodes of $\mathrm{SpO}_{2}<95 \%$, ventilator positive inspiratory pressure, interface or tubing air leakage, $\mathrm{CO}_{2}$ retention, ventilator settings, and MI-E use are considered. Patients are also taught air stacking and manually assisted coughing.

Postextubation, the therapists, nurses, and in particular, the family and personal care attendants provide MI-E via oral-nasal interfaces up to every 30 minutes until $\mathrm{SpO}_{2}$ no longer dips below 95\% and the patients are clear of secretions. This can be critical both because the burden can be excessive for critical care staff and because the home care providers need to become very proficient in both MI-E and CNVS for the patient to go home safely from the critical care unit and to avoid future episodes of ARF. When postextubation oral intake is

Table 2 Extubation criteria for unweanable, ventilator-dependent patients

\begin{tabular}{l} 
Criteria \\
Afebrile and normal white blood cell count \\
Carbon dioxide tension of $40 \mathrm{mmHg}$ or less at peak inspiratory \\
pressures less than $30 \mathrm{~cm} \mathrm{H}_{2} \mathrm{O}$ on full ventilatory support and \\
physiologic breathing rate, as needed \\
Oxyhemoglobin saturation $\geq 95 \%$ for 12 hours or more in ambient air \\
All oxyhemoglobin desaturations below $95 \%$ reversed by mechanical \\
insufflation-exsufflation via translaryngeal tube \\
Fully alert and cooperative, receiving no sedative medications \\
Chest radiograph abnormalities cleared or clearing \\
Air leakage via upper airway sufficient for vocalization upon cuff deflation \\
\hline
\end{tabular}

unsafe, gastrostomies can be placed without intubation, as described earlier.

Data were reported on 157 consecutive "unweanable" patients: 25 with SMA, 20 with DMD, 16 with ALS, 51 with other NMDs, 17 with spinal cord injury, and eleven with polio. Eighty-three who refused tracheostomies were transferred from other hospitals. These patients could not pass spontaneous breathing trials before or after extubation. Once $\mathrm{SpO}_{2}$ was maintained at $95 \%$ or higher in ambient air, the patients were extubated to CNVS and frequent MI-E. Extubation success was defined as not requiring reintubation during the hospitalization. Before hospitalization, $96(61 \%)$ patients had no experience with NVS, 41 (26\%) used it part-time, and 20 (13\%) were CNVS-dependent. First-attempt protocol extubation success rate was 95\% (149 patients). All 98 extubation attempts on patients with assisted CPF $160 \mathrm{~L} /$ minute or higher were successful. Six of eight patients who initially failed extubation succeeded on subsequent attempts, so only two bulbar ALS patients with no measurable assisted CPF underwent tracheotomy. ${ }^{30}$ Long-term chronic care facilities and nursing facility ventilator units lack the expertise to admit these patients, and we have found discharge directly home to always be preferable even when no ventilator weaning is possible.

\section{Decannulation of unweanable patients}

In 1996, we reported the decannulation of 50 unweanable patients with neuromuscular weakness. ${ }^{5}$ Earlier, in 1990 and 1991 we and others reported the routine decannulation of high-level traumatic SCI patients to CNVS. ${ }^{10,15}$ The principles of decannulating unweanable patients are essentially the same as those for extubation. Any ventilator-dependent patient whose bulbar-innervated musculature is adequate such that saliva aspiration does not cause a continuous decrease in baseline $\mathrm{SpO}_{2}$, and with assisted CPF of $120 \mathrm{~L} /$ minute or higher, is a candidate for decannulation to NVS. Patients with tracheostomy tubes who had no CTMV-free breathing ability with VCs of $250 \mathrm{~mL}$ or greater invariably wean from continuous support after decannulation. Many wean to nocturnal-only NVS within 3 weeks of decannulation. Tube removal also facilitates speech and swallowing. Thus, the lack of all ventilator-free breathing ability in SCI does not mandate tracheostomy or phrenic or diaphragm pacing. Only severe glottis dysfunction that results in aspiration of saliva with baseline oxyhemoglobin saturation below $95 \%$ does. $^{48}$ All decannulated patients in one study preferred CNVS to CTMV for convenience, speech, swallowing, cosmesis, comfort, safety, and overall. ${ }^{49}$ 


\section{Conclusion}

A simple evaluation designed to assess a patient's respiratory muscle function rather than a full battery of pulmonary function tests designed for obstructive/intrinsic lung disease, and the application of pressures to the body and airways to support inspiratory and expiratory muscle function rather than supplemental oxygen therapy and bronchodilators, can permit many progressively weakening patients to avoid ARF and tracheotomies. Those who do develop ARF, are intubated, and cannot pass spontaneous breathing trials can, nevertheless, be extubated to full-setting CNVS and MI-E. Quality of life is optimized by NVS. Unfortunately, the conventional paradigm is that anyone who requires aroundthe-clock respiratory support needs a tracheostomy tube. Thus, an entirely different evaluation and treatment paradigm is required for the optimal and humane management of patients who have primarily respiratory muscle weakness, rather than lung disease, as was ascertained by the April 2010 consensus of clinicians from 22 centers, reporting on 760 CNVS-dependent patients. ${ }^{50}$ Although no controlled studies are needed to demonstrate that patients without any autonomous ability to breathe require ventilatory support to survive, controlled studies have now demonstrated significantly greater survival by noninvasive, rather than invasive, ventilatory support for DMD patients and unanimous overall patient preference for noninvasive, rather than invasive, management for all neuromuscular diagnoses. ${ }^{45,49} \mathrm{It}$ is, therefore, time for a paradigm shift from invasive to noninvasive management of these patients.

\section{Acknowledgments}

Patient consent was obtained for the use of the patient photos.

\section{Disclosure}

The authors report no conflicts of interest in this work.

\section{References}

1. Bach JR, Bianchi C, Aufiero E. Oximetry and indications for tracheotomy for amyotrophic lateral sclerosis. Chest. 2004;126(5):1502-1507.

2. Bach JR, Gupta K, Reyna M, Hon A. Spinal muscular atrophy type 1: prolongation of survival by noninvasive respiratory aids. Pediatr Asthma Allergy Immunol. 2010;22(4):151-162.

3. Bach JR. Amyotrophic lateral sclerosis: prolongation of life by noninvasive respiratory AIDS. Chest. 2002;122(1):92-98.

4. Bach JR, Alba AS. Management of chronic alveolar hypoventilation by nasal ventilation. Chest. 1990;97(1):52-57.

5. Bach JR, Saporito LR. Criteria for extubation and tracheostomy tube removal for patients with ventilatory failure. A different approach to weaning. Chest. 1996;110(6):1566-1571.

6. Kang SW, Bach JR. Maximum insufflation capacity. Chest. 2000;118(1): $61-65$.
7. Currie DC, Munro C, Gaskell D, Cole PJ. Practice, problems and compliance with postural drainage: a survey of chronic sputum producers. Br J Dis Chest. 1986;80(3):249-253.

8. Williams AJ, Yu G, Santiago S, Stein M. Screening for sleep apnea using pulse oximetry and a clinical score. Chest. 1991;100(3):631-635.

9. Bach JR, Alba AS, Bodofsky E, Curran FJ, Schultheiss M. Glossopharyngeal breathing and noninvasive aids in the management of post-polio respiratory insufficiency. Birth Defects Orig Artic Ser. 1987;23(4):99-113.

10. Bach JR. New approaches in the rehabilitation of the traumatic high level quadriplegic. Am J Phys Med Rehabil. 1991;70(1):13-19.

11. Dail C, Rodgers M, Guess V, et al. Glossopharyngeal Breathing. Downey, CA: Rancho Los Amigos Department of Physical Therapy; 1979.

12. Dail CW, Affeldt JE. Glossopharyngeal Breathing [video]. Los Angeles, CA: Department of Visual Education, College of Medical Evangelists; 1954

13. Webber B, Higgens J. Glossopharyngeal breathing: what, when and how? [video]. West Sussex, UK: Aslan Studios Ltd; 1999.

14. Bach JR, Bianchi C, Vidigal-Lopes M, Turi S, Felisari G. Lung inflation by glossopharyngeal breathing and "air stacking" in Duchenne muscular dystrophy. Am J Phys Med Rehabil. 2007;86(4):295-300.

15. Bach JR, Alba AS. Noninvasive options for ventilatory support of the traumatic high level quadriplegic patient. Chest. 1990;98(3): 613-619.

16. Bach JR, Kang SW. Disorders of ventilation : weakness, stiffness, and mobilization. Chest. 2000;117(2):301-303.

17. Bach JR, Mahajan K, Lipa B, Saporito L, Goncalves M, Komaroff E. Lung insufflation capacity in neuromuscular disease. Am J Phys Med Rehabil. 2008;87(9):720-725.

18. Bach JR, Baird JS, Plosky D, Navado J, Weaver B. Spinal muscular atrophy type 1: management and outcomes. Pediatr Pulmonol. 2002;34(1):16-22.

19. Bach JR, Alba AS, Shin D. Management alternatives for post-polio respiratory insufficiency. Assisted ventilation by nasal or oral-nasal interface. Am J Phys Med Rehabil. 1989;68(6):264-271.

20. Bach JR, Alba AS. Intermittent abdominal pressure ventilator in a regimen of noninvasive ventilatory support. Chest. 1991;99(3):630-636.

21. Bach JR, Robert D, Leger P, Langevin B. Sleep fragmentation in kyphoscoliotic individuals with alveolar hypoventilation treated by NIPPV. Chest. 1995;107(6):1552-1558.

22. Allen J. Pulmonary complications of neuromuscular disease: a respiratory mechanics perspective. Pediatr Respir Rev. 2010;11(1):18-23.

23. Ishikawa Y. Manual for the Care of Patients using Noninvasive Ventilation. Matsudo, Japan: Japan Planning Center; 2005.

24. Bach JR, Alba AS, Saporito LR. Intermittent positive pressure ventilation via the mouth as an alternative to tracheostomy for 257 ventilator users. Chest. 1993;103(1):174-182.

25. Richards GN, Cistulli PA, Ungar RG, Berthon-Jones M, Sullivan CE. Mouth leak with nasal continuous positive airway pressure increases nasal airway resistance. Am J Respir Crit Care Med. 1996;154(1): 182-186.

26. Bach JR, Gonzalez M, Sharma A, Swan K, Patel A. Open gastrostomy for noninvasive ventilation users with neuromuscular disease. $A m J$ Phys Med Rehabil. 2010;89(1):1-6.

27. Bach JR. Noninvasive respiratory management of high level spinal cord injury. J Spinal Cord Med. 2012;35(2):72-80.

28. Gomez-Merino E, Bach JR. Duchenne muscular dystrophy: prolongation of life by noninvasive ventilation and mechanically assisted coughing. Am J Phys Med Rehabil. 2002;81(6):411-415.

29. Guérin C, Bourdin G, Leray V, et al. Performance of the CoughAssist insufflation-exsufflation device in the presence of an endotracheal tube or tracheostomy tube: a bench study. Respir Care. 2011;56(8): 1108-1114.

30. Bach JR, Gonçalves MR, Hamdani I, Winck JC. Extubation of patients with neuromuscular weakness: a new management paradigm. Chest. 2010;137(5):1033-1039. 
31. Fishburn MJ, Marino RJ, Ditunno JF. Atelectasis and pneumonia in acute spinal cord injury. Arch Phys Med Rehabil. 1990;71(3):197-200.

32. Garstang SV, Kirshblum SC, Wood KE. Patient preference for inexsufflation for secretion management with spinal cord injury. J Spinal Cord Med. 2000;23(2):80-85.

33. Bach JR, Smith WH, Michaels J, et al. Airway secretion clearance by mechanical exsufflation for post-poliomyelitis ventilator-assisted individuals. Arch Phys Med Rehabil. 1993;74(2):170-177.

34. Barach AL, Beck GJ. Exsufflation with negative pressure; physiologic and clinical studies in poliomyelitis, bronchial asthma, pulmonary emphysema, and bronchiectasis. AMA Arch Intern Med. 1954;93(6): 825-841.

35. Bach JR. Mechanical insufflations-exsufflation. Comparison of peak expiratory flows with manually assisted and unassisted coughing techniques. Chest. 1993;104(5):1553-1562.

36. Waldhorn RE, Herrick TW, Nguyen MC, O’Donnell AE, Sodero J, Potolicchio SJ. Long-term compliance with nasal continuous positive airway pressure therapy of obstructive sleep apnea. Chest. 1990;97(1): 33-38.

37. Bach JR. Conventional approaches to managing neuromuscular ventilation failure. In: Bach JR, editor. Pulmonary Rehabilitation: The Obstructive and Paralytic Conditions. Philadelphia, PA: Hanley \& Belfus; 1996:285-301.

38. Bach JR, Rajaraman R, Ballanger F, et al. Neuromuscular ventilatory insufficiency: effect of home mechanical ventilator use v oxygen therapy on pneumonia and hospitalization rates. Am J Phys Med Rehabil. 1998;77(1):8-19.

39. Toussaint M, Steens M, Wasteels G, Soudon P. Diurnal ventilation via mouthpiece: survival in end-stage Duchenne patients. Eur Respir J. 2006;28(3):549-555.

40. Bach JR, Tuccio MC, Khan U, Saporito LR. Vital capacity in spinal muscular atrophy. Am J Phys Med Rehabil. 2012;91(6):487-493.
41. Schroth MK. Special considerations in the respiratory management of spinal muscular atrophy. Pediatrics. 2009;123 Suppl 4:S245-S249.

42. Kohler M, Clarenbach CF, Böni L, Brack T, Russi EW, Bloch KE. Quality of life, physical disability, and respiratory impairment in Duchenne muscular dystrophy. Am J Respir Crit Care Med. 2005;172(8):1032-1036.

43. McKim DA, Griller N, LeBlanc C, Woolnough A, King J. Twenty-four hour noninvasive ventilation in Duchenne muscular dystrophy: a safe alternative to tracheostomy. Can Respir J. 2013;20(1):e5-e9.

44. Villanova M, Brancalion B, Mehta AD. Duchenne muscular dystrophy: life prolongation by noninvasive ventilatory support. Am J Phys Med Rehabil. In press.

45. Ishikawa Y, Miura T, Ishikawa Y, et al. Duchenne muscular dystrophy: survival by cardio-respiratory interventions. Neuromuscul Disord. 2011;21(1):47-51.

46. Mills B, Bach JR, Zhao C, Saporito L, Sabharwal S. Posterior spinal fusion in children with flaccid neuromuscular scoliosis: the role of noninvasive positive pressure ventilatory support. $J$ Pediatr Orthop. 2013;33(5):488-493.

47. Mahajan KR, Bach JR, Saporito L, Perez N. Diaphragm pacing and noninvasive respiratory management of amyotrophic lateral sclerosis/ motor neuron disease. Muscle Nerve. 2012;46(6):851-855.

48. Bolikal P, Bach JR, Goncalves M. Electrophrenic pacing and decannulation for high-level spinal cord injury: a case series. $J$ Spinal Cord Med. 2012;35(3):170-174.

49. Bach JR. A comparison of long-term ventilatory support alternatives from the perspective of the patient and care giver. Chest. 1993;104(6): 1702-1706.

50. Bach JR, Gonçalves MR, Hon A, et al. Changing trends in the management of end-stage neuromuscular respiratory muscle failure: recommendations of an internal consensus. Am J Phys Med Rehabil. 2013;92(3):267-277.
Journal of Neurorestoratology

\section{Publish your work in this journal}

The Journal of Neurorestoratology is an international, peer-reviewed, open access online journal publishing original research and review articles on the subject of Neurorestoratology. To provide complete coverage of this revolutionary field the Journal of Neurorestoratology will report on relevant experimental research, technological advances, and

\section{Dovepress}

clinical achievements. The manuscript management system is completely online and includes a very quick and fair peer-review system, which is all easy to use. Visit http://www.dovepress.com/testimonials.php to read real quotes from published authors. 